\title{
Color Naming Experiment in Mongolian Language
}

\author{
Nandin-Erdene Osorjamaa (Corresponding author) \\ The National University of Mongolia, Orkhon school, \\ Department of Translation Studies, Mongolia \\ E-mail: nandiaosorjamaa@gmail.com,nandinerdeneosorjamaa@yahoo.com \\ Nansalmaa Nyamjav \\ School of Science, Faculty of Humanities, Department of European Studies \\ The National University of Mongolia, Mongolia \\ E-mail: nansalmaa@mail.ru
}

Received: 20-03- 2015

Accepted: 21-06- 2015

Advance Access Published: August 2015

Published: 01-11- 2015

doi:10.7575/aiac.ijalel.v.4n.6p.58

URL: http://dx.doi.org/10.7575/aiac.ijalel.v.4n.6p.58

\begin{abstract}
There are numerous researches on color terms and names in many languages. In Mongolian language there are few doctoral theses on color naming. Cross cultural studies of color naming have demonstrated Semantic relevance in French and Mongolian color name Gerlee Sh. (2000); Comparisons of color naming across English and Mongolian Uranchimeg B. (2004); Semantic comparison between Russian and Mongolian idioms Enhdelger O. (1996); across symbolism Dulam S. (2007) and few others. Also a few articles on color naming by some Mongolian scholars are Tsevel, Ya. (1947), Baldan, L. (1979), Bazarragchaa, M. (1997) and others. Color naming studies are not sufficiently studied in Modern Mongolian. Our research is considered to be the first intended research on color naming in Modern Mongolian, because it is one part of Ph.D dissertation on color naming. There are two color naming categories in Mongolian, basic color terms and non- basic color terms. There are seven basic color terms in Mongolian. This paper aims to consider how Mongolian color names are derived from basic colors by using psycholinguistics associative experiment. It maintains the students and researchers to acquire the specific understanding of the differences and similarities of color naming in Mongolian and English languages from the psycho-linguistic aspect.
\end{abstract}

Keywords: modern Mongolian, basic color terms, non-basic color terms, associative experiment, signal word, response word, color naming

\section{Introduction}

According to Berlin and Kay’s (1969), color terms are divided into two sets; ${ }^{1}$

The basic color terms are:

(a) not subsumed under other terms---for instance, crimson and scarlet are not basic terms in English, since they are varieties of red. But orange is a basic term, since it is not subordinate to any other color term;

(b) morphologically simple---terms such as bluish, bluish green and chocolate-colored, even golden, are excluded;

(c) not collocationally restricted---for example, blond, which describes only hair, is

not a basic color term;

(d) frequently used---rare words such as puce, and technical words such as xanthic,

are excluded. (Taylor, 1995: 8)

The non-basic color terms exhibit one or more of the following properties:

(a) they are not monomorphemic, that is they are polymorphemic;

(b) they are not frequently used;

(c) they are specific varieties of some other color; or

(d) they are collocationally restricted to a certain kind of substance.

Through Berlin and Kay's (1969) experiments, it was found that the same colors tended to be picked out by subjects when they are asked to find the best example of their basic color terms, despite the fact that different languages have different numbers of basic color terms and the boundaries of the basic color categories of different languages do not coincide ${ }^{2}$.

\footnotetext{
${ }^{1}$ Evelynne, Ki-mei Mui (1998) Some aspect of Cantonese color terms

${ }^{2}$ Kay and McDaniel (1978) and Taylor ( 1995)
} 
According to Williams J.N (2006) Associationism maintains that all knowledge is represented in terms of associations between ideas, that complex ideas are built up from combinations of more primitive ideas, which, in accordance with empiricist philosophy, are ultimately derived from the senses.

Like SIGNAL WORD - RESPONSE WORD module in associative method of psycho- linguistics, there are many studies that are proven to be an experiment; any optional word can form color collocations. This kind of experiment on color terms in Mongolian has not been made to date.

It is the main reason that the psycho-linguistic study of specified color names to determine thinking pattern and ways of Mongolian people.

\section{Experimental procedure}

A questionnaire for the experiment - according to SIGNAL WORD - RESPONSE WORD model

1. Please, give the answers for all the questions and help us with our study.
1. Age: .......
2. Gender: ......
3. Education:
4. Nationality:
5. Job:
6. Place of birth: ......
7. Residence/ year:

2. You can write Response words as many as you can

1. Tsagaan /White/....

2. Khar /Black/....

3. Ulaan $/$ Red/.

4. Shar/Yellow/.

5. Nogoon /Green/

6. Tsenkher/Blue/.

7. Khukh /Blue/.

8. Yagaan $/$ Pink/....

9. Bor $/$ Brown $/ .$.

10. Khuren /Light brown/

11. Saaral /Grey/....

12. Busad ungu/Other colors/

In the experimental assignment, in order to find the alternative opportunity for 11 color indicated names which were the basis of Berlin and Kay (Berlin and Kay, 1969)'s study, as well as to reconnoiter what words to be given, the participants were provided with the opportunity to choose the words on their own and asked to write "other colors", so, in total 12 words to be responded. Based on the explanations which are alternatives for 11 color indicated words (Berlin and Kay, 1969), 11 of which do not match with the Mongolian equivalents to some extent, for instance: the English word blue has two different meanings in Mongolian /khukh and tsenkher/ as well as the English word "brown", whereas different in English from each other words "violet" and "pink" have the same meaning in Mongolian. Therefore, it should be noted that the list of 11 words employed in our study varies from the list made by the above-mentioned researchers to some extent.

The participants of the experiment could write as many response words for one signal word, which can be considered as the method to develop chain (denoted by descriptive color words). The list of colors we offered: white, black, red, yellow, blue, blue, green, pink, brown, brown, grey and "other colors".

11 color terms by Berlin and Kay (1969) are: white, black, red, yellow, green, blue, brown, grey, orange, purple, pink

Aged 16-74, with the professions related to 11 different sectors, 150 male and 150 female, total of 300 participants were involved in this experiment and were asked to fill out the questionnaire within 20-30 minutes in order to determine what words and attributives associate with the 11 colors and provided with chance to widen their choice by adding the $12^{\text {th }}$ color.

\begin{tabular}{|c|c|c|c|c|c|c|}
\hline № & Model & $\begin{array}{l}\text { Number of } \\
\text { participants }\end{array}$ & Age & Gender & Task & Time \\
\hline 1 & $\begin{array}{l}\text { Signal word } \longrightarrow \\
\text { Response word }\end{array}$ & 300 & $16-74$ & $\begin{array}{l}\text { male } 150 \text {, female } \\
150\end{array}$ & $\begin{array}{l}\text { Write } \\
\text { responses to } \\
\text { the signal } \\
\text { word }\end{array}$ & $20-30 \mathrm{~min}$ \\
\hline
\end{tabular}

The main significance of the association chain method is to provide with chance to determine as many words as the participant can respond for a one SIGNAL WORD.

Our method includes the possibility of choosing as many words for a one signal word, thus, the participants needed more time, and defining a certain color by associating it with a color indicated name also requires more time. So, having 10 minutes for the questionnaire is insufficient and it becomes a proof for reasons given. Thus, we gave 20-30 minutes for this experiment.

In order to identify modern Mongolians' specifics of color description tendency, we focused on the results to be accurate and chose the people who have not resided in a foreign country, were out of foreign language influence, have 
the Mongolian way of thinking and are conscious about the Mongolian language. Moreover, age, sex, ethnicity, place of birth and residence, profession and occupation, education level and other biological and social factors were taken into account.

Except those factors, our study is aimed to classify the color terms, observe the response words of the participants, and determine the ways of associating color and how these words are formed in modern Mongolian in the example of color "Shar" (yellow).

\section{Results}

402 responses were given for 11 signal words by 300 participants. Number of response words by an each signal word and its frequency are presented below.

Response words and frequency obtained by the experiment using signal word - response word method

\begin{tabular}{llcc}
\hline № & Signal word & Number of response words & Frequency \\
\hline 1 & Shar (yellow) & 65 & 596 \\
2 & Tsagaan (white) & 50 & 731 \\
3 & Khar (black) & 45 & 448 \\
4 & Nogoon (green) & 40 & 568 \\
5 & Ulaan (red) & 32 & 506 \\
6 & Saaral (grey) & 32 & 438 \\
7 & Yagaan(pink, violet) & 29 & 464 \\
8 & Tsenkher (light blue) & 28 & 490 \\
9 & Khukh (blue) & 28 & 448 \\
10 & Khuren (light brown) & 27 & 387 \\
11 & Bor (brown) & 26 & 361 \\
& & 402 & 5437 \\
\hline
\end{tabular}

Besides giving 11 color indicated words, we separately added "other colors" in order to test whether participants add any other variations to the list of the words we offered or was there something naturally determined. The result indicated that 17 names (single name, color indicated collocation) with frequency of 64 were given for signal word "other colors". And, about 70 per cent of the participants did not give a response word and for this point we can conclude that not giving a response is also can be a response too. Absence of a word in their vocabulary may be related to the lack of their general knowledge, difficulties of naming or impossibility to differ one from other color indicated words. Judging by the responses given significant percentage were the response words coincided with "other colors", therefore we focused on the response words for the 11 signal words.

The color "SHAR" (YELLOW) got the largest number of response words with high frequency (300 participants gave 65 types of responses for this color with frequency of 596) which suggests that the Mongolians color terms through their association with many attributes of their lifestyle and habitation as well as it is a proof that life itself colored with many of them. The most repeated words were golden yellow, bamboo yellow, autumn leaf like yellow, sandy yellow, coin yellow which shows the links of the Mongolians with nature and natural colors play important role in their cognition. Based on the documents, 153 people out of 300 found response word "golden yellow" which suggests that the Mongolians associate yellow color with the gold. As the word "gold" is defined in a dictionary, it is the root element of chemistry and yellow colored precious metal, which matches with our results. If we consider that word "root" means the "beginning of any substance", we can understand why color indicated collocation "golden yellow" comes first in to mind of the Mongolians. Furthermore, we cannot deny of possibility of symbolic meaning due to naming the sun as golden yellow.

There were many response words with the lowest frequency or given by single participant, so we think the explanation for each word could be different. It is because besides their feelings, the researchers themselves are the part of a language and cultural group with cognition. Based on this point, we can conclude that the words "coin yellow" "cattle dung yellow", "sedimentary yellow", "robe yellow", "straw yellow" be the reflection of our traditional nomadic lifestyle and explain it by the fact that cognitive experience of the Mongolians is common. On the other hand, the words "fire yellow", "creamy yellow". "apricot yellow", "lemon yellow", "pumpkin yellow", "honey yellow", "flax yellow" or brownish-yellow, narcissus yellow or bright yellow, saffron flower yellow", "poppy yellow" came in our life later as the reflection of urbanized life and are familiar with the foreign culture and mostly used by the young people. The word "cattle dung yellow" relates to the Mongolian traditional husbandry when yellow associates with the color of cattle's manure, "robe yellow" with the color of the flared robe wore by the monks. Among the response words were many which belong to the plants as "straw yellow" /dried straw and stems of the plants/, "flax yellow" /plant used for 
making ropes and extracting oil/, "poppy yellow" / grassy plant with red and yellow flowers/, "saffron yellow" /medicinal plant with orange flower/, "narcissus yellow" /plant with mainly yellow flower/; the rest of the names except the "straw yellow" are not so familiar for the public.

There were some response words with low frequency but of interest, therefore it is required to review further. For instance: there is a likelihood that they were influenced by the foreign culture such as "honey yellow, "peach yellow" and others, related to the fruits which were given by those who live in urban cities.

The ways of establishing response words for color "yellow", its association and attributives.

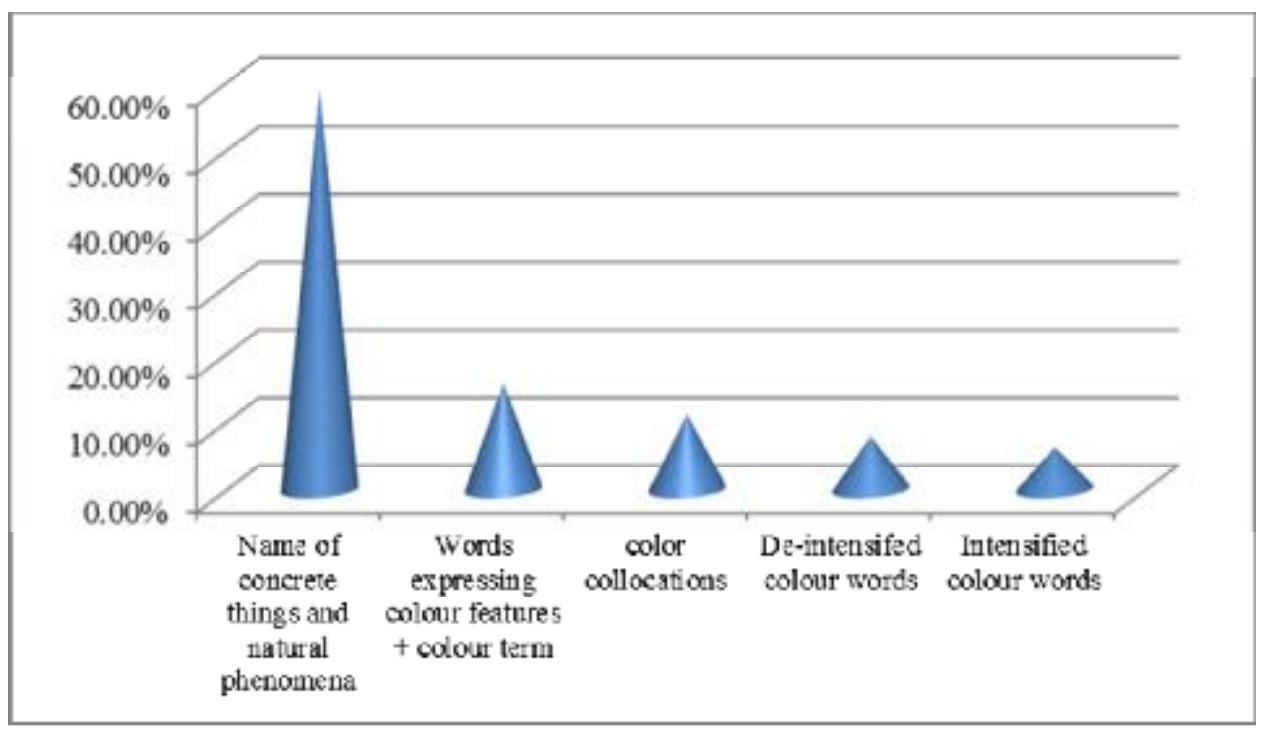

Figure 1. The way of thinking on forming yellow color in Mongolian

From the diagram, it is obvious that the most effective method of all ways of thinking on forming yellow color indicated words is relating it to the items (concrete things) such as golden yellow, bamboo yellow, straw yellow, creamy yellow, autumn leaf yellow, baby stool yellow. Then follows another method of collocation formed by adding (de-intensified) adjectives color, for example: "yellowish", "blurry yellow", "greyish yellow". Then goes the method of forming collocation by the model "color word + color word" as red yellow, green yellow, white yellow and orange yellow whereas the method of using intensified and de-intensified adjectives had the lowest efficiency. From all, the most effective was the way of forming color indicated words by relating them to the color of items and was thoroughly indicated in the pie chart.

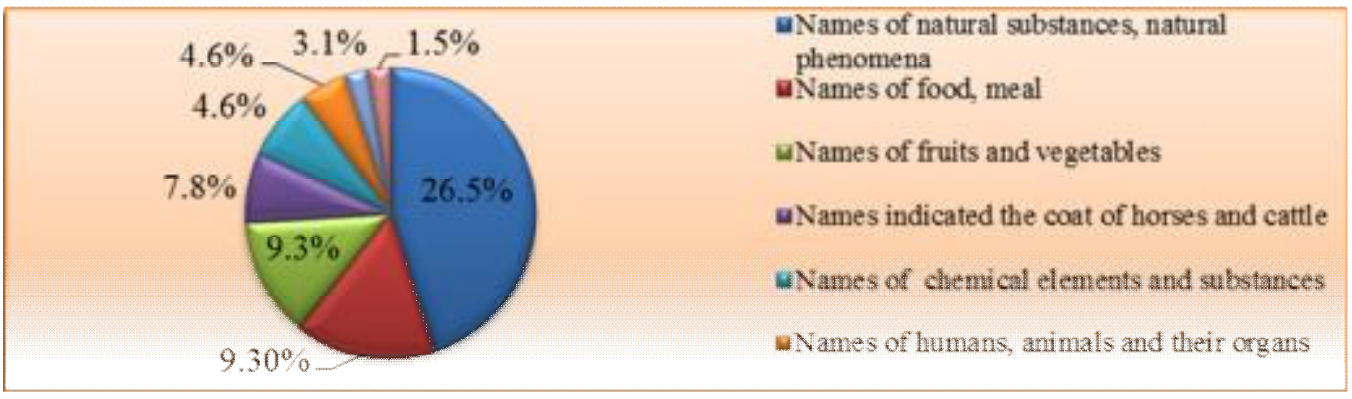

Figure 2. Ways of forming collocations through associating with "yellow" with the names of concrete things and phenomena.

The response words given by the participants:

A. Names of natural substances, natural phenomena + color indicated name: snow white (184), ash grey (131), fire red (67), grassy green (47), sky blue (20) etc.

B. Names of food, meal + color indicated name: milky white (64), creamy yellow (13), chocolate brown (13), oily brown (8) etc. This type of response words originated from the dairy products and shows the links with traditional culture of the Mongolians.

C. Names of fruits and vegetables + color indicated name: raisins violet (92), orange yellow (12), apple red (11), peach yellow (9), lemon yellow (2), apricot yellow (1) etc. There are many words in Mongolian associated with the names of fruits; however less of which are related to vegetables. For instance: beetroot, 
pumpkin, apricot colored which could be borrowed from translation experience, are not common for the Mongolians.

D. Names indicated the coat of horses and cattle: pure white like sheep (21), reddish pink (11), reddish yellow with white mane and tale /used for naming color of stallion/ (2), pale brownish /used for naming horse color/ (1) and pinto horse color show the strong links with nomadic lifestyle and culture

E. Names of chemical elements and substances + color term: golden yellow (153), ashy black (43), silver grey (6), brass yellow (4), zinc white (2), copper red (1).

F. Names of humans, animals and their organs + color term: swan white, conch white (21), liver violet (18), bone white (16), blood red (16), baby camel brown (3), egret white (3), sheep white (1), calf black (1), demonstrates also the strong and close connections of the Mongolians with the cattle they breed.

G. Names of items + color term: paper white (39), ink violet (34), scarf like blue (13), porcelain white (8), ink blue (6). These words are related to the items used in our everyday life and show the close links with the house-holding.

H. Names of precious stones + color term: lazurite blue /khukh/ (105), lazurite green (101), pearly white (94), lazurite blue /tsenkher/ (2), turquoise green (35), nacreous white (20), coral red (6)

- Words expressing color features + color term: blurry green (34), pale white (11), flabby yellow (5), and creamy yellow (4).

In Mongolian some appendixes linking with the color indicated words can make collocations in connection with a few color indicated words or single color indicated word.

- Color collocation: black blue (121), brown /close to chestnut brown/ (63), purple (60)8 brown (54 black green (43), brown red (41), red pink (38) and etc. suggest that when people want to name intermediate colors they address mainly to the colors.

- De-intensified color words: brownish (57), yellowish (20), bluish (7), reddish (6), pinkish (5), yellowish yellow (4), yellowish brown (2) and etc.

- Intensified color words: very brown (114), berry white (111), very red (108), very blue (104), very black (95). These words are created by adding prefixes "pad", "tas", "tsal" and "tsel" to a certain color word, and called non-free collocation. For example: prefix "tas" can be added only to word black and cannot be connected to other words. The same situation is observed with other words.

Except the above-mentioned units there were some words with no less frequency and introduced in Mongolian language lately, originated from foreign cultures (came in to mind of the Mongolians from the foreign cartoons) such as Unitel /cellphone communication provider/ green (23), Oriflame /cosmetics brand/ green (6), Shrek green (6), fuchsia pink (2); these words proves the reflection of the changes in life on our language.

The participants formed in total 402 color indicated words with frequency of 5437 by SIGNAL WORD-RESPONSE WORD model. Except proving the experiment results suggested in Chapter 2 based on the methods, models, structure and other sources, it was defined that the most effective way of forming color indicated collocations is the model of "item, phenomena's name + color indicated name". Naming the items and phenomena by its color within this method is considered to be the main way of thinking of the Mongolians to form the color words.

\section{Conclusion}

To conclude, Berlin and Kay's (1969)'s study 11 basic colors in English do not match with Mongolian color terms.

We aimed to involve people with various ages, genders, places of birth and residence, educations and occupations, out of foreign languages and cultures in order to identify their way of thinking based on their response words for "yellow"; we find although Mongolian have common features they have different ways of forming the color names depending on age, sex, places of residence and occupation.

- High frequency response words are the words which characterize the thinking of the Mongolians; they are plausible, similar to each other in the terms of our mentality and with possibility to establish a group with the close meaning words. The lower frequency indicates the likelihood of foreign cultures influence and expresses an individual's personal cognitive experience Thus; it can be subject to further studies.

- The way how Mongolians related yellow color to natural phenomena, meals, naming the horses by its color (coat) has become the reflection of the Mongolians' thinking (mentality) and express its main husbandry and way of thinking.

\section{References}

Baldan, L. (1979). Uran zohiold ungunii neriig heregleh tuhai asuudald, (Color names in Mongolian literature), Ulaanbaatar.

Bazarragchaa, M. (1997). Mongolchuud umni ungu zusiig herhen nerledeg ve? (How Mongolians name things using color terms), Ulaanbaatar.

Berlin, B. and Kay, P. (1969). Basic Color Terms: Their Universality and Evolution, Berkeley, University of California Press. 
Dulam, S. (2007). Ungiin belgedel, zug chigiin belgedel, (Color Symbolism), $2^{\text {nd }}$ edition, Ulaanbaatar.

Enhdelger, O. (1996). Semantic comparison between Russian and Mongolian idioms. Moscow.

Gerlee, Sh. (2000). Semantic relevance in French and Mongolian color name. Ulaanbaatar.

Kay, P. and McDaniel, C. K. (1978). The linguistic significance of the meanings of basic color terms. Language, 54, 610-46.

Mui E. Kim. (1998). Some aspects of color terms in Cantonese. SOAS, ms.

Taylor, J.R. (1995). Linguistic Categorization. Oxford, Clarendon Press.

Tsevel, Ya. (1947). Mongolchuudiin ungu erhemleh yos (Color preference among Mongolians).

Uranchimeg, B. (2004). Comparisons of color naming across English and Mongolian. Ulaanbaatar.

Williams, J.N. (2006). Associationism and Connectionism. Cambridge, UK, p 559. 